\title{
Imagining a Borderless Future: Methodologies for Multimodal Remix in Pan-terrestrial People's Anthem
}

\author{
ANDREW DEMIRJIAN \\ Assistant Professor, Hunter College
}

\begin{abstract}
Flags and national anthems, as symbols of the nation state, frequently rely on music, lyrics and visual design to enlist and manipulate emotional bonds. Can these same tools be used, alternatively, to disentangle connections to our concepts of country, notions of borders or even the idea of the nation state itself? This is the question at the core of Pan-terrestrial People's Anthem, an interdisciplinary remixing of the lyrics and music of 195 countries' national anthems and their corresponding flags to create a body of poetry, music and videos. In an era of increasing closeddoor nationalism, this article proposes that remix strategies can be used to unravel our concepts of nations, which traditionally magnify differences between countries and overemphasize a false sense of uniqueness, and to point instead to the interconnectedness between populations. In this context, remix strategies become a model for a future imaginary, a world that emphasizes the interdependence of beings and spaces that transcend established geopolitical boundaries. Through the case study of Pan-terrestrial People's Anthem, I argue that remix strategies are particularly well suited as an aesthetic structural tactic for engaging with pressing issues where intertwined and entangled futures are at stake.
\end{abstract}

In an era of increasing closed-door nationalism, where people are held stateless in camps at various borders, this article proposes that remix strategies may open paths to disrupt traditional constructs of the nation state as fixed and permanent, resulting in one where borders are more permeable. Flags and national anthems, as symbols of the nation state, frequently rely on music, lyrics and visual design to strengthen emotional bonds. Can these same tools be used, alternatively, to disentangle connections to our concepts of country, notions of borders or even the idea of the nation state itself? This is the question at the core of Pan-terrestrial People's Anthem, an interdisciplinary remixing of the lyrics and music of 195 countries' national anthems and their corresponding flags to create a body of poetry, music and videos. ${ }^{1}$ Taken collectively, this work seeks to dismantle the extractive and exploitative structures that have dominated geopolitical frameworks and to conceive instead of formations that privilege interconnectivity and cooperation.

In this context, remix strategies become a model for a future imaginary, a world that emphasizes the interdependence of beings and spaces that transcend established geopolitical boundaries. 
Through the case study of Pan-terrestrial People's Anthem, I argue that remix strategies are particularly well suited as an aesthetic structural tactic for engaging with pressing issues where our intertwined and entangled futures are at stake.

The germination of this work was in response to the global rise of nationalism and its incongruence with the hybrid, diasporic, intersectional sensibility of contemporary culture. In this environment, I began interweaving fragments of lyrics, musical phrases of anthems and flag graphics as an analogy for the complex histories of border movement that make up much of our modern identities. In the field of electronic literature, practices that combine texts, sound, imagery, videos and interaction are referred to as multimodal. ${ }^{2}$ Pan-terrestrial People's Anthem uses these multimodal crossings between artistic disciplines as a metaphor for traversing geospatial borders. Remix is compelling as an artistic practice, because it mirrors the associative and combinatorial aspect of our cognition. We are constantly making connections between bodies of knowledge, borrowing a concept from one domain and putting it in dialogue with another. In the same way that Hugo Munsterberg pointed to the cognitive process of attention as being visualized in the film language of the close-up, I want to suggest that remix reflects the associative leaps and recombinatory links of thought. ${ }^{3}$

The juxtaposition of associations in remix can also function as a distancing effect to see media and popular culture from different perspectives. In his essay titled The Editor, Stefano Basilico discusses artists who work with preexisting material. He states, "They don't need to shoot the films for they understand that the editing and shaping of what may already exist in the world is a more powerful and revealing act. Furthermore, by selecting what already exists, they foreground the deconstructive nature of this act, whose purpose is to destroy illusion and reveal the inherent manipulative powers of the medium."4 Although he is discussing artists who work with film, the same may be said for artists working in multimodal remix. Relocating material and changing its context can illuminate the political aims of its messaging. Perhaps an anthem that is built entirely from preexisting material, that foregrounds and questions the histories and structures of the past, is a less illusionistic choice for the future.

But where to begin? Working with remix can often engender an intimidating feeling similar to the dread of the blank page but arising, instead, from too much information. How to work with an overwhelming amount of sonic, visual and linguistic material? What form should the work take? How to bridge the different technologies? It can be daunting to stare at 27,523 words and hundreds of minutes of music that can be parsed and recombined in limitless ways. What can give work coherence and allow for creative entry into the process?

Paradoxically, constraint systems can lead to creative freedom. For the purposes of this article, a constraint system is the application of a set of rules that refines or reduces the totality of a collection of data through a particular framework, filter or pattern that enables a different way of seeing, reading or hearing a collection of elements. For instance, in Doug Aitken's Hysteria (2010), the viewer sees excerpts of film and video of audiences from rock concerts going into trance states; however, we never see the band that is performing. By creating a constraint system that filters concert footage to show only the audience cut-away shot, removing the primary interest of the original source material - the musicians - the work transforms the original footage from a document about a band's performance to a study of gestures of fandom, ecstatic states and the transformative power of music. In the article "Remix and the Dialogic Engine of Culture," Martin Irvine writes, "With its ever-accruing encyclopedia of music resources and intertextual relations, 
jazz exemplifies a form of practiced dialogism that opens up the deeper underlying generative processes in other cultural forms." In the spirit of the dialogic call and response in remixing, I will map out the methodologies for multimodal remixing that I use in Pan-terrestrial People's Anthem, in the hope that these concepts may be recombined and remixed by others.

\section{THIS MUST BE THE PLACE}

One methodological approach can be based on the location from which an excerpt is taken within a time-based work. For example, track seven in Pan-terrestrial People's Anthem employs a process where the last two musical notes of dozens of anthems (typically a plagal cadence), are strung together consecutively, creating the feeling of an endless ending, an overdose of grandiose crescendos. Using this technique gives listeners a sense of the uniformities among many anthems whose intent, one would imagine, is to be as unique as possible. By hearing these two chord fragments one after another in a long sequence, the listener develops a heightened attention to little changes and curious similarities between dozens of scores, and this creates a feeling that the composers are trying to outdo each other in a sonic pomposity competition. One striking observation when working with so many anthems is how countries that struggled for independence from their colonial oppressors in the 1950s and 1960s use the sonic language of the colonizer for their new anthem. The pieces seem to mimic the instrumentation, harmonies and arrangements of the oppressor in order to validate themselves.

A methodology that emphasizes location in the sonic domain can also be applied to the linguistic domain. In a video for this same track, the text on the lower third of the screen displays a sequence of the last lyrical phrases from dozens of the anthems over their two chords of music. Lyrical lines like "Victoriously dispel the strife of darkness" or "Hold out a welcome for one and all" are presented sequentially on screen every five seconds. This remix presents the songs' concluding words in dialogue with each other, a succession of parting thoughts written decades apart from across the globe.

As an alternative to working with precise locational information, location could also be determined by chance. Graphically, these specific lyrical and musical constraint systems are visually combined with chance techniques using algorithmically generated flags in the video. The new, hybrid flags are created by randomly choosing several national flags from the database and combining them by choosing iconography, color and shape to build a new form (Figure 1). For instance, the sun icon from the Argentinian flag known as the Sun of May and the four stars of the Chinese flag may be combined with Brazil's yellow diamond and Namibia's red diagonal stripe, all layered together in one new composite image. Freeing up a portion of the design to be algorithmically generated allows for non-human intervention in the work; chance operations and algorithmic ignorance of geopolitical histories can make flags with unexpected graphic combinations that humans, informed by local, regional and global lived experience might not consider. 


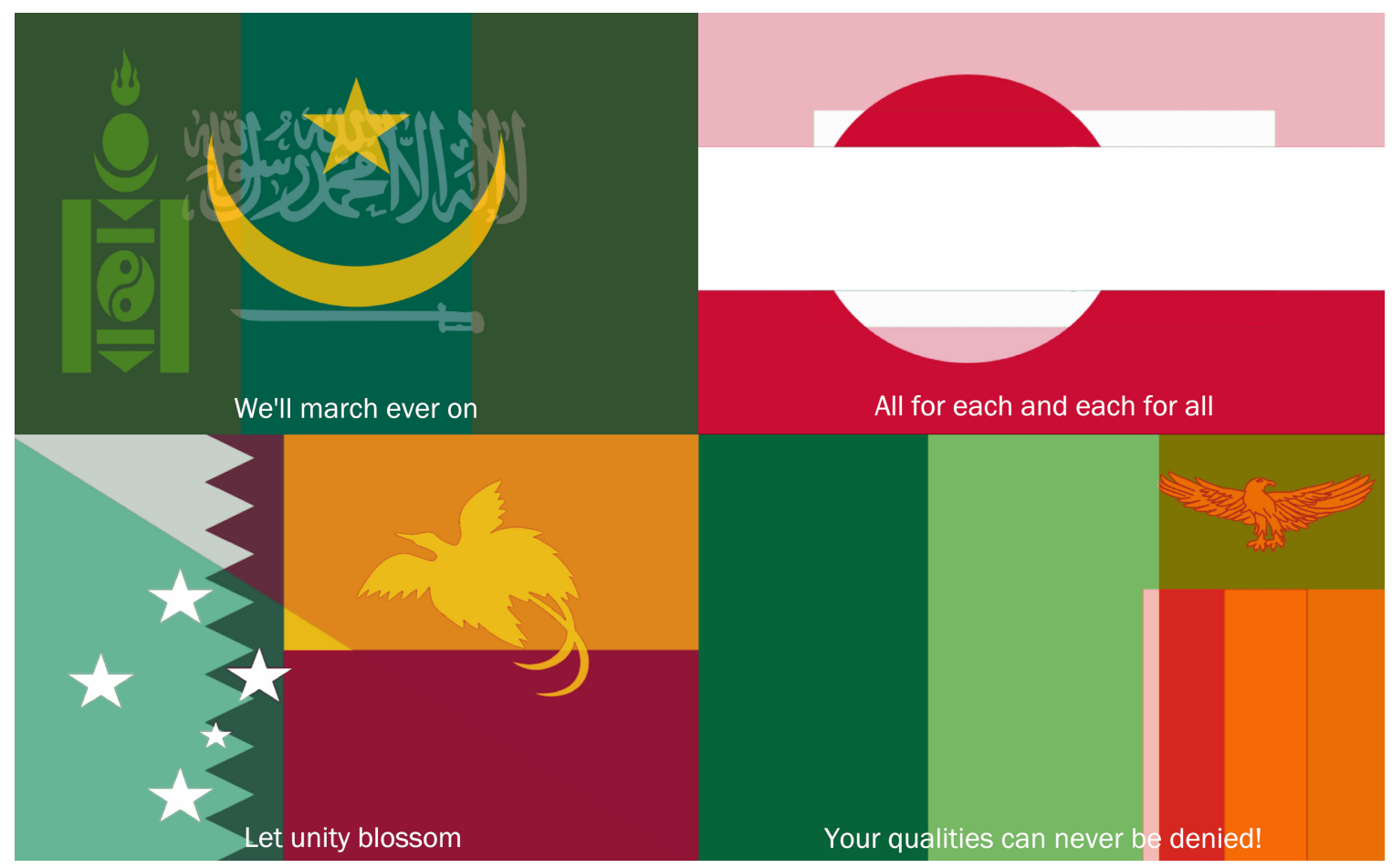

Figure 1. Stills from Pan-terrestrial People's Anthem track 7.

\section{RHYTHM NATION}

Another way to approach a multimodal remix is to study the material based on its sonic characteristics. Working with the shared sonic qualities of language and music can allow for arrangements of material that provide alternative connections between sources, eliding the dominance of semantic approaches. A Python script and Natural Language Toolkit Library were used to search the phonetic content of all the words in the anthem lyrics to find moments of alliteration in adjacent words like "bombs bursting" and "breezes blow." In track three of Panterrestrial People's Anthem, these percussive sounding textual combinations were paired with moments from the anthems where only percussion was playing, a song made entirely of national anthem drum samples. The video for this composition is a data visualization by artist James Proctor that creates a map based on the location of letters in the alphabet for each alliterative word combination. A small edition artist book published by Contour Editions features some of the most frequent alliterative combinations by letter, which are then placed into shapes that resemble countries on a map. However, instead of being any one particular country, the perimeters of multiple countries are layered on top of one another and traced around the outer edge, creating a new land mass.

Multimodal remixing enables two or more constraint systems to operate simultaneously in an artwork on the visual, linguistic or sonic registers. To create a sense of unity when using multiple constraints, it can be helpful to have a meta-constraint that connects them in a thematic or formal way. In the example above, there is a "meta-rule" of rhythmic beat that connects the rapid pulse from the verbal domain of alliterative lyrics to the percussive attacks in the non-verbal sonic 
domain. This framework can provide focus to a piece that brings together different sensory material, thus creating a more cohesive whole.

Unlike the previously mentioned process of finding alliterative moments in the linguistic domain, there were no programming libraries that could easily identify solo percussion sounds within a song. The desire to find these isolated drumming parts led to listening to all 195 audio tracks while taking notes on the sonic characteristics of each piece, from the individual song's key to its instrumentation and other novel identifying characteristics. Aesthetically, I believe much can be gained by using computational analysis of a database in conjunction with the lived experience of a human sensory encounter as a form of knowledge about the material qualities of a database.

While the methods are very different in their approach, both forms of understanding can highlight aspects of the content that can't be found in the other. Programming has a particular goal in mind: it is written to search explicitly for a targeted output as efficiently as possible. For instance, the alliterative search referenced above scours the database for each word's first phoneme. If the next word's first phoneme matches that of the prior word, then it gets added to the list of alliterative words. However, in the humanly executed algorithm of experiencing the database (also known as listening), a search for something specific like isolated drum sounds may lead down a meandering path toward identifying other sonic characteristics that can then shape the work in a previously unimagined way.

Track five illustrates this experience. While performing focused listening for isolated drums, I became aware of how many trite solo synthesizer renditions of anthems existed. This eventually led to the creation of track five, a mashup of pure synth versions of anthems. Unlike most of the other tracks, which involved painstaking sampling and selection of notes and melodies and orchestrating them in particular locations, this track applied a different method. Here, the tempos and keys were aligned for multiple songs, and then for each new measure a different track was randomly selected. The raw, unfussy approach coupled with the prepackaged synth tones creates a looseness that is unlike other tracks on the album. A hybrid approach that combines computationally derived insights with human ear/hand/eye sensorial perceptions, although painstaking, can provide variation and surprise that can encourage sustained engagement.

\section{BETTER TOGETHER}

This next methodology includes processes that involve collaborations between people and between people and algorithms. Track eight of Pan-terrestrial People's Anthem starts with a snowball poem, a type of poem that was developed by the OULIPO group, known for creating rule-based literary works beginning in the early 1960s. The poem takes a form where each successive word in the poem is one character longer than the prior word (see Figure 2). To generate these poems in Panterrestrial People's Anthem, the 27,523 words in the lyric database were arranged in groups of one letter words, two letter words, three letter words, etc. Then using a Python script, hundreds of tenline snowball poems were generated where each new line grew by one additional word that was one letter longer than the previous word. Visually, the poem forms a sloping curve of language to the right as it moves down the page. Scouring the output looking for striking combinations of words or uncommon uses of language can be liberating and can distance the author from their own cognitive perspective to allow for inspiration from algorithmically chosen language. 


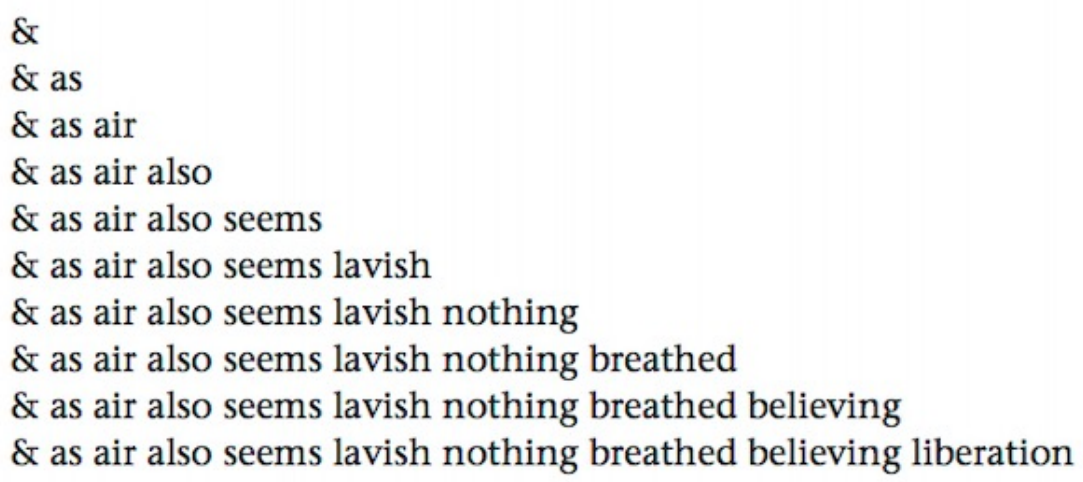

Figure 2. Snowball poem from Pan-terrestrial People's Anthem.

These finished poems were then given to the vocalist Ami Yamasaki, my collaborator on this track, for her to interpret in a recording studio. In contrast to the sonic qualities of an anthem sung by thousands in a sports arena, she created a very intimate interpretation that feels more suited for being sung to oneself on a walk in the woods or sitting in a park. The methodological approach here is one of filtering and exchanges among computational, textual and sonic domains until the poem is ready to be spatialized.

\section{SPACE IS THE PLACE}

One of the most intriguing new areas for remix methodologies is in the spatial affordances of emerging technologies. In the creation of Pan-terrestrial People's Anthem, I was thinking about ways to embrace themes of shifting borders or borderlessness, to underscore that the concept of a nation state was relatively new and that borders are not only artificial but are often in flux. To support this concept, several of the compositions for the album are available as YouTube VR videos where the mix of the audio track can be in constant movement, using Ambisonics, a form of audio playback that simulates a $360^{\circ}$ sound environment. The video playback of $360^{\circ}$ films on YouTube is an Ambisonics audio track that is mapped to a binaural playback on headphones. As the listener moves their phone or clicks and drags their video, the sounds pan and change based on their positioning, creating a formal element that underscores thematic ideas. The sonic effect creates movement of instruments in the aural field with no official fixed resting place, conceptually connecting this movement to the idea of creating geospatial structures without hard border edges.

Additionally, the sonic content of this track is joined visually with an algorithmically generated still composite image of global parades. The visuals suggest blurring spaces without strict geospatial boundaries; participants, banners and props from a Malaysian Merdeka Parade, an Eyo Iga Etti procession in Lagos and a Saint Patrick's Day parade in Boston may gradually merge and blend together in a hybrid image that is wrapped onto $360^{\circ}$ of space. The images in these environments also serve as markers of location to better orient the user to their place as they move their mobile device or mouse in circular space. 


\section{CONCLUSION}

Artists who work with remix often find inspiration through creating constraints for filtering large databases of material. ${ }^{6}$ For this article, I have attempted to map out some possible trajectories for continuing in this vein with a heightened attention to multimodal remixing among textual, sonic and visual domains. I've sketched out four areas that have potential for further exploration, but many more possible trajectories exist. What other "meta-rules" can be conceived that can bring multiple domains together in surprising and generative ways? How can collaborative "hand-offs" between artists working in different disciplines or artists and algorithms generate new multimodal possibilities? What might be other scenarios for mapping spatial coordinates from textual and sonic domains that support thematic concerns?

In the post-pandemic world, right-wing groups and their financial supporters will certainly use COVID-19 as a way to stoke fear and further tighten borders, limit cultural exchange and inhibit contact with others. However, if the pandemic has shown us anything, it is how inextricably interconnected we all are. This may be the time to more seriously consider rethinking geopolitical spaces and setting aside the troubled concept of the nation state, extractive mindsets and relentless global competition and to imagine alternative possibilities of cooperative existence. Pan-terrestrial People's Anthem proposes soundscapes to these possible future spaces.

\section{ENDNOTES}

1. Pan-terrestrial People's Anthem, Contour Editions, August 28, 2018, https://www.contoureditions.com/releases/ce.onl_0022/ce.onl_0022.html

2. N. Katherine Hayles, "Electronic Literature: What is it?" The Electronic Literature

Organization, 2 January 2007, https:/eliterature.org/pad/elp.html.

3. Hugo Munsterberg, "The Photoplay: A Psychological Study," Project Gutenberg, accessed June 5, 2020, https://www.gutenberg.org/ebooks/15383.

4. Stefano Basilico, "The Editor," in Cut: Film as Found Object in Contemporary Video, ed., Stefano Basilico (Milwaukee: Milwaukee Art Museum, 2004), 30.

5. Martin Irvine, "Remix and the Dialogic Engine of Culture," in The Routledge Companion to Remix Studies, eds., Eduardo Navas, Owen Gallagher, and xtine burrough (Milton Park, Abingdon, Oxon: Routledge, 2014), 21.

6. Eran Hadas, Creativity in Constraint, Immerse.news, 19 November, 2018. https://immerse.news/digital-storytelling-is-constantly-evolving-and-as-such-it-keepsquestioning-its-forms-b19e6fd08c6.

\section{REFERENCES}

Basilico, Stefano. "The Editor." In Cut: Film as Found Object in Contemporary Video. Edited by Stefano Basilico, 28-45. Milwaukee: Milwaukee Art Museum, 2004.

Hadas, Eran. "Creativity in Constraint.” Immerse. November 19, 2018.

https://immerse.news/digital-storytelling-is-constantly-evolving-and-as-such-it-keepsquestioning-its-forms-b19e6fd08c6. 
Hayles, N. Katherine. "Electronic Literature: What is it?" The Electronic Literature Organization. January 2, 2007. https://eliterature.org/pad/elp.html.

Irvine, Martin. "Remix and the Dialogic Engine of Culture." In The Routledge Companion to Remix Studies. Edited by Eduardo Navas, Owen Gallagher, and xtine burrough, 15-42. Milton Park, Abingdon, Oxon: Routledge, 2015.

Munsterberg, Hugo. “The Photoplay: A Psychological Study.” Project Gutenberg. Accessed June 5, 2020. https://www.gutenberg.org/ebooks/15383.

\section{AUTHOR BIO}

Andrew Demirjian is an interdisciplinary artist who works with remix, rhythm and ritual. His installations, performances and videos playfully upend cultural obsessions of self-improvement, optimization and efficiency. Demirjian creates unconventional environments for critical reflection where layers of language and sound shape space. He is currently a Fellow at the MIT Open Documentary Lab, where he is working on a computational text analysis project for an interactive installation.

Demirjian's work has been exhibited at The Museum of the Moving Image, Eyebeam, Fridman Gallery, Transformer Gallery, Rush Arts, The Center for Book Arts, The Newark Museum and many other galleries, festivals and museums. The MacDowell Colony, Puffin Foundation, Artslink, Harvestworks, Diapason, The Experimental Television Center, The Bemis Center, LMCC Swing Space, The Visual Studies Workshop and the New Jersey State Council on the Arts are among of the organizations that have supported his work. He teaches theory and production courses in emerging media in the Film and Media Department and the Integrated Media Arts MFA program at Hunter College. 